\title{
Studying Manometric Anatomy to Understand Structural Heart Disease at the Organ Scale
}

\author{
Alice F. Liang1', Valeria Mezzano², Alejandra Leo-Macias², Esperanza Agullo Pascual' ${ }^{2}$, Chris Petzold ${ }^{1}$, \\ Kristen Dancel-Manning ${ }^{1}$, Gregory Morley ${ }^{2}$, and Mario Delmar ${ }^{2}$. \\ ${ }^{1 .}$ Microscopy Laboratory, DART/New York University Langone Health, New York, USA. \\ 2. Leon H Charney Division of Cardiology, Department of Medicine/New York University Langone \\ Health, New York, USA.
}

The heartbeat originates from thousands of cells contracting coordinately. Complex molecular network organization between cardiomyocytes allows their prompt and efficient interaction. Cardiac intercellular junctions, known as intercalated discs (ID) play major roles in cardiac excitability and structural stability of cardiac tissue. Intercalated discs are composed of three types of cell junctions: fascia adherens, desmosomes and gap junctions. Loss of molecular organization of the ID can lead to cardiomyopathies that affect the heart pump function and/or lead to cardiac arrhythmias. The mechanisms responsible for these arrhythmias remain poorly understood. One example of such a disease is arrhythmogenic cardiomyopathy (AC; also known as 'arrhythmogenic right ventricular cardiomyopathy' or 'ARVC') which is an important cause of sudden cardiac death in the young [1]. AC is a genetic cardiomyopathy where most of the disease-causing mutations occur in proteins that form part of the desmosome such as plakophilin-2 (PKP2). The relationship between PKP2 and connexin 43 (Cx43), a primary component of gap junctions is known as key in the pathogenesis of AC.

Modern methods of light and electron microscopy allows us to better visualize the ID, and precisely localize its related proteins. We characterized the ultrastructural features of PKP2-heterozygous-null (PKP2-Hz) murine hearts and explored the relation between PKP2 abundance, $\mathrm{Na}_{\mathrm{v}} 1.5$ the pore-forming subunit of the sodium channel, and cardiac electrical synchrony [2]. Tomographic images of wild type and PKP2-Hz mice revealed sporadic loss of desmosomes, non-uniform and enlarged intercellular membrane space of the PKP2-Hz mice (Fig. 1). Our studies also showed that the siRNA-mediated loss of PKP2 expression affects the amplitude and kinetics of the sodium current, and PKP2 is required for transcription of genes that control calcium cycling and cardic rhythm [3]. Better preserved ultrastructure using high-pressure freezing and freeze-substitution coupled with electron tomography indicated that the gap junction plaque can make contact with the mitochondrial outer membrane, and inner part of a desmosome [4]. Since desmosomes and gap junctions are clearly defined under transmission electron microscope, it is hard to believe that molecules of desmosomes and gap junctions would physically interact. By combining single molecule imaging technology, correlative light and electron microscopy and 3D EM, we demonstrated that the outer edge of Cx43 crosstalks with PKP2 clusters, and the dimension of these clusters depend on Ankyrin G expression (Fig. 2). We also demonstrated that $\mathrm{Na}_{\mathrm{v}} 1.5$ molecules are major contributors to cardiac sodium current, and loss of $\mathrm{Na}_{\mathrm{v}} 1.5$ expression reduces intercellular adhesion strength.

Acute and chronic illness often leads to fibrotic scarring. Fibrosis in the heart can occur secondary to myocardial infarction, chronic heart failure or genetic cardiomyopathies. The fibrotic process leads to non-cardiac cell population infiltration. An increase in these populations, such as occurs during disease, will change tissue architecture and affect intercellular communication giving rise to a proarrhythmic substrate. However, ultrastructural descriptions of non-cardiac cells during fibrosis is lacking. Nanoscale 
3D reconstruction of murine cardiac scar tissue using a mouse model of ventricular ablation was performed using serial block face SEM (SBFSEM). Three main cell types were identified: fibroblasts, endocardial cells and inflammatory cells (likely macrophages). The 3D model of all cells in the dataset showed mouse ventricular ablation scar tissue has an embedded cellular network, previously uncharacterized, where every cell connects to more than two other cells through intercellular contacts. A cellular network such as this may provide the substrate for electrical communication between healthy cardiac muscle and scar tissue.

\section{References:}

[1] SR Kaplan et al, Heart Rhythm, 1(1) (2004), p.3.

[2] M Cerrone et al, Cardiovascular Research, 95(4) (2012), p460.

[3] M Cerrone, J Montnach, X Lin et al, Nature Communications., 8(1) (2017), p.106.

[4] M Delmar and FX Liang, Heart Rhythm, 9(5) (2015), p.835.
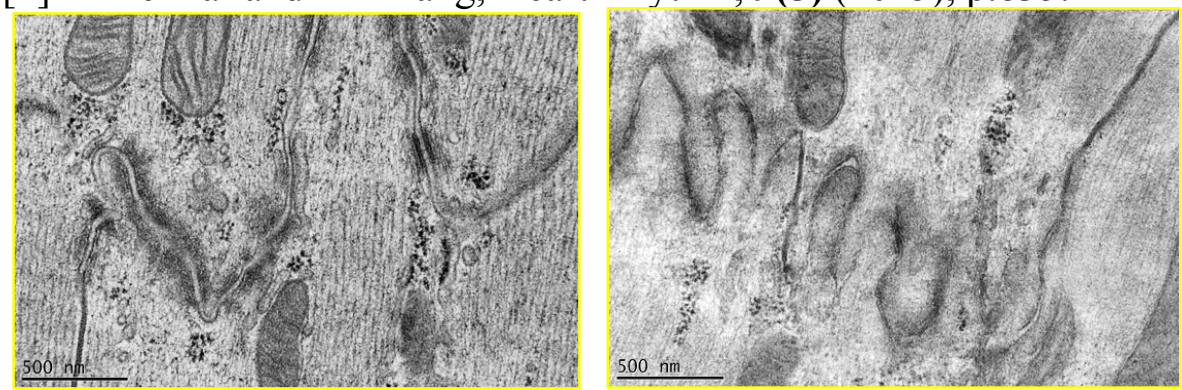

Figure 1. Structure difference of intercalated disc of wild type (left) vs. pkp2-Hz (right) mouse.
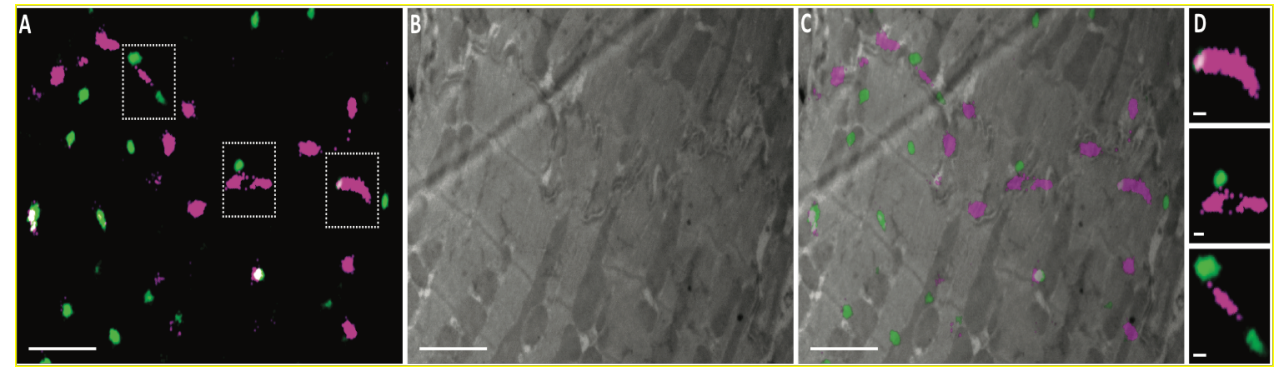

Figure 2. Correlation of Nav1.5 (green) and N-cadherin (purple) at intercalated disc.

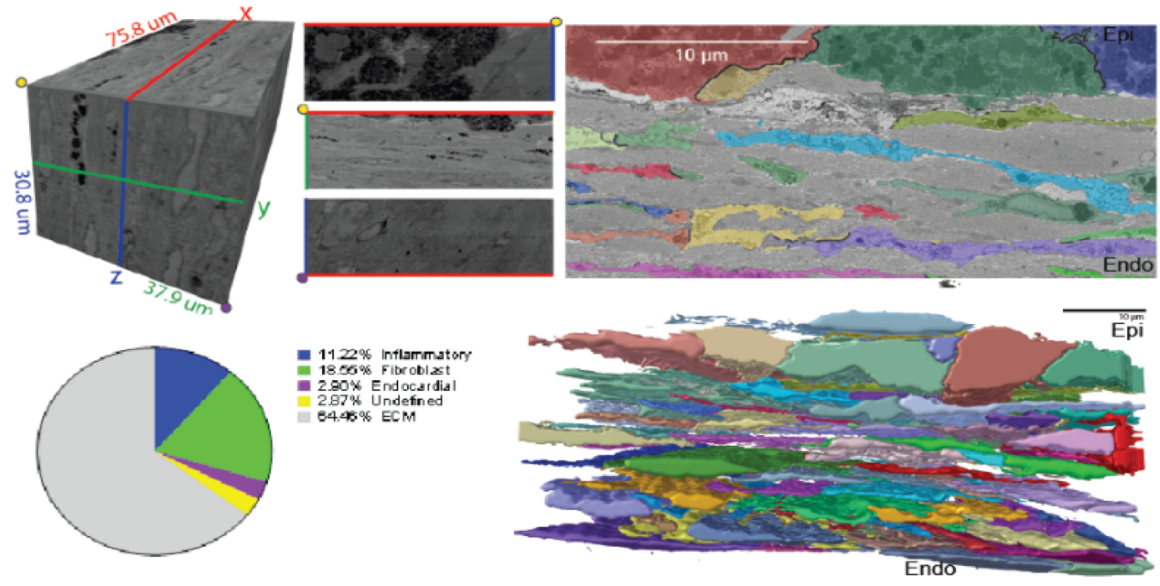

Figure 3. Cell and network characteristics of myocardial scar tissue. 